\title{
Enhanced tensile ductility and strength of electrodeposited ultrafine-grained nickel with a desired bimodal microstructure
}

\author{
Qian Zhang ${ }^{\mathrm{a}, \mathrm{b}}$, Ying Liu ${ }^{\mathrm{a}, \mathrm{b}, *}$, Yongsheng Liu ${ }^{\mathrm{a}, \mathrm{b}}$, Yanhong Ren ${ }^{\mathrm{a}, \mathrm{b}}$, Yanxia Wu ${ }^{\mathrm{a}}$, Zhipeng Gao ${ }^{\mathrm{c}}$ \\ Xiaolei $\mathrm{Wu}^{\mathrm{d}}$, Peide $\mathrm{Han}^{\mathrm{a}, \mathrm{b}}$ \\ a College of Materials Science and Engineering, Taiyuan University of Technology, 030024 Taiyuan, China \\ ${ }^{\mathrm{b}}$ Key Laboratory of Interface Science and Engineering in Advanced Materials, Taiyuan University of Technology, 030024 Taiyuan, China \\ ${ }^{c}$ Institute of Applied Mechanics \& Biomedical Engineering, College of Mechanics, Taiyuan University of Technology, 030024 Taiyuan, China \\ d State Key Laboratory of Nonlinear Mechanics, Institute of Mechanics, Chinese Academy of Sciences, Beijing 100190, China
}

\section{A R T I C L E I N F O}

\section{Keywords:}

Electrodeposition

Nickel

Bimodal microstructure

Twins

Spatial distribution

Mechanical property

\begin{abstract}
A B S T R A C T
This work aims to use surfactant-assisted direct current electrodeposition technique to prepare four types of bimodal nickel, under different current densities. Bimodal Ni is obtained with different grain size and spatial distribution of CG and UFG areas showing a big disparity in mechanical properties. As a result of small population of coarse-grained surrounded by quite a lot of ultrafine-grained forming a unique shell-and-core bimodal structure, bimodal one present the best comprehensive mechanical properties with an ultrahigh tensile strength $(\sim 847 \mathrm{MPa})$ and a considerable plastic strain $(\sim 16.7 \%)$. Deformation initial, bimodal structures display more positive strain hardening to meaningful strains than unimodal structure of UFG and CG. Particularly bimodal one work-hardening rate is the highest thanks to its structure (UFG occupy $76.7 \%$ in total number fraction) and the distribution of growth twins. Growth twins in this article are referred to $\Sigma 3(111)$ coherent twins playing an important role in improving high strength, enhancing uniform plastic deformation ability.
\end{abstract}

\section{Introduction}

Scientists and engineers have, for centuries, focused on obtaining materials with both high strength and ductility. This can be attributed to the problem that strength and ductility are mutually exclusive [1-16]. High-strength materials are needed, particularly in addressing current challenges, such as environmental pollution, global warming, and energy crisis. Strong materials reduce the weight of vehicles, thereby improving their energy efficiency. In industrial processes, good ductility is needed to prevent catastrophic failures. Nanocrystalline (NC, $d_{\text {grain }}<100 \mathrm{~nm}$ ) and ultrafine-grained (UFG, $100 \mathrm{~nm}<$ $\left.\mathrm{d}_{\text {grain }}<1 \mu \mathrm{m}\right)$ metal materials exhibit high strength. However, their ductility, particularly the uniform elongation in tension, is quite low and, in most cases, nowhere closes to that of normal metals [17]. This drawback has been an insurmountable hurdle in bringing NC/UFG materials into industrial application.

Previous studies reported that the poor ductility of NC and UFG metal materials primarily result from the restrain of dislocation-mediated mechanism in small grain size, leading to the low strain-hardening capability. As a result, the appearance of early necking can induce the materials' fracture, which can be attributed to the lack of limitation to plastic instability. A variety of strategies, such as the multi- and bimodal microstructures, nanolaminated structure, and gradient nanostructure, aimed at improving the poor ductility of NC and UFG materials have been reported $[8,18,19]$. Among them, the bimodal grain size distribution microstructure, in which volume fraction of micron-sized grains are introduced into the NC and UFG matrix, is considered as an efficient strategy.

Strength-to-ductility ratio strongly depends on grain size distribution [20-22]. For instance, experimental evidence presented by $\mathrm{Wu}$ et al. demonstrated that bimodal grain size distribution could balance increased strength and high ductility in metal materials [18]. One strategy suggested to enhance the ductility of NC/UFG materials is to process a bimodal grain structure, in which the fine grains provide strength and the coarse grains improve ductility. Zhao et al. used cryomilling and, subsequently, quasi-isostatic forging processes (before the famous Ceracon forging was developed) to prepare the bimodal UFG/coarse-grained (CG) Ni. Average grain size of the bimodal Ni was $2.1 \mu \mathrm{m}$, yielding at $312 \mathrm{MPa}$ with $49 \%$ ductility [7]. Tao Qian et al. prepared the bimodal UFG/CG Ni through severe plastic deformation and annealing technologies. With the increase of the volume fraction of CG, the ultimate tensile strength (UTS) of the specimen decreased from

\footnotetext{
* Corresponding author at: College of Materials Science and Engineering, Taiyuan University of Technology, 030024 Taiyuan, China

E-mail address: liuying01@tyut.edu.cn (Y. Liu).
} 

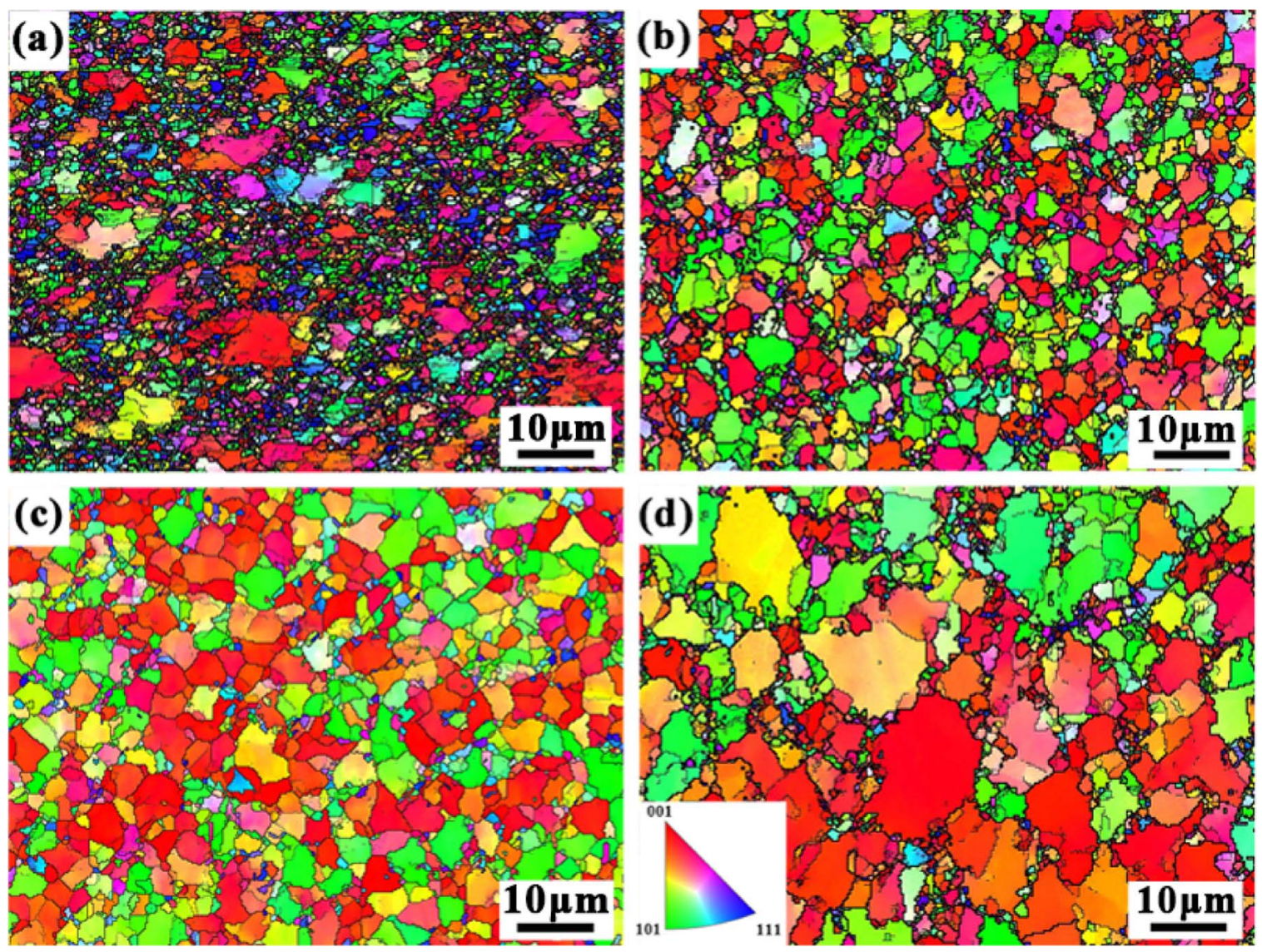

Fig. 1. EBSD images of different samples: (a), (b), (c), (d) show the microstructure of bimodal one, two, three and four.

$470 \mathrm{MPa}$ to $380 \mathrm{MPa}$, and uniform elongation increased from $1.5 \%$ to $20 \%$ [23]. These results reveal that the plastic instabilities can be overcome by the additional strain-hardening capability of micron-sized grains, increasing the tensile ductility while minimizing loss of strength. However, some problems persist. First, the bimodal grain size distribution microstructure is obtained from two- or multistep processes accompanied by secondary recrystallization, and the annealing process is usually difficult to control because of different influencing parameters. Second, only a narrow area fraction of micron-size grained aggregate was investigated during the heat treatment. Despite enormous efforts in the past decade, quantitative understanding of the grain size, spatial distribution, and the mechanical behaviors of bimodal Ni remains poor. The present investigation is undertaken with the specific objective of preparing high-performance Ni with desired bimodal grain size distribution through a simple method and evaluating microstructural changes occurring during the tensile process. Herein, bimodal $\mathrm{Ni}$ with a different grain size distribution has been obtained through direct current electrodeposition in an improved traditional electroplating bath. The microstructure, mechanical properties, and deformation mechanism of the as-synthesized samples are investigated and discussed.

\section{Experimental}

\subsection{Preparation of the bimodal $\mathrm{Ni}$}

The dense and bulk bimodal Ni was synthesized through direct current electrodeposition, with a focus on adjusting the current density and the type of additives. All reagents used were analytical grade, and deionized water was utilized to prepare the plating bath. The electrolyte consisted of $\mathrm{NiSO}_{4} \cdot 6 \mathrm{H}_{2} \mathrm{O}(208 \mathrm{~g} / \mathrm{L}), \mathrm{NiCl}_{2} \cdot 6 \mathrm{H}_{2} \mathrm{O}(30 \mathrm{~g} / \mathrm{L}), \mathrm{H}_{3} \mathrm{BO}_{3}$ $(30 \mathrm{~g} / \mathrm{L})$, and a small amount of sodium dodecyl benzene sulfonate. The
$\mathrm{pH}$ of the solution was adjusted to 4.0-4.2 through diluted hydrochloric acid, and the temperature was controlled at $55^{\circ} \mathrm{C}$. To eliminate the effect of impurities further, the electrolyte was first purified at a low current density of $0.1 \mathrm{~A} / \mathrm{dm}^{2}$ for two days. The bulk Ni with a thickness of approximately $0.28 \mathrm{~mm}$ was synthesized on the austenitic stainless steel sheets $(40 \mathrm{~mm} \times 50 \mathrm{~mm} \times 1 \mathrm{~mm})$ under the direct current density ranging from $0.35 \mathrm{~A} / \mathrm{dm}^{2}$ to $1.12 \mathrm{~A} / \mathrm{dm}^{2}$. Then, all samples were peeled off from the stainless steel substrate.

\subsection{Microstructure characterizations}

The crystallographic structure of the as-deposited bulk Ni samples was investigated through X-ray diffraction (XRD, D/max 2500PC, CoK $\alpha$ radiation, $\lambda=0.179 \mathrm{~nm}$ ) with a step of $0.05^{\circ}$. Microstructure and grain size distribution of the specimens were characterized under an electron back scatter diffraction (EBSD) using a TSL OIM system on a Philips XL30 FEG SEM with step sizes of $0.25 \mu \mathrm{m}$. The EBSD samples were vibratory polished using diamond suspension, and a final particle size of $0.2 \mu \mathrm{m}$ was achieved, then electropolished through an etchant of $30 \mathrm{vol} \%$ nitric acid and $70 \mathrm{vol} \%$ ethanol for several seconds at $20 \mathrm{~V}$ and room temperature.

\subsection{Mechanical property tests}

Mechanical property tests were composed of tensile tests and Vickers hardness. The dog-bone-shaped specimens with a gauge crosssection of $2.5 \mathrm{~mm} \times 0.28 \mathrm{~mm}$ and a gauge length of $14.0 \mathrm{~mm}$ were cut from the as-deposited bimodal bulk Ni sheet using a wire-cut electrical discharge machine, and then the surface was polished to a mirror-like finish. The tensile tests were carried out on an Instron 5582 testing machine at a strain rate of $1.2 \times 10^{-4} \mathrm{~s}^{-1}$. Moreover, three identical specimens were measured to ensure sufficient experimental data are 
obtained. The fracture surface morphology of as-electrodeposited $\mathrm{Ni}$ samples was observed through a scanning electron microscope (ZEISS EVO18). The hardness variation in different bimodal structures was studied through depth sensing microindentation at room temperature. A Vickers diamond indenter, with a load of $490.3 \mathrm{mN}$ maintained for $15 \mathrm{~s}$, was used in microindentation study of bimodal Ni. Nearly fifteen indentations were taken under the same sample.

\section{Experimental results}

\subsection{Microstructural features}

The fabricated microstructure morphologies of the samples are studied through EBSD. The EBSD images are shown in Fig. 1. All the samples, several of which were prepared under the current density of $0.35,0.70,0.86$, and $1.12 \mathrm{~A} / \mathrm{dm}^{2}$, showed bimodal structure characteristics composed of UFG and CG. As shown, the grain size and spatial distribution of UFG and CG are significantly different. In Fig. 1a, the amount of UFG is significantly higher than that of CG. A small quantity of CG is tightly surrounded by large amounts of UFG with a thickness of a few micrometers. This is called the bimodal one. The number of UFG in Fig. 1b is significantly less than that in Fig. 1a. Bimodal two has a similar structure with that of bimodal one; however, in bimodal two, only a couple layers of UFG surround CG. As shown in Fig. 1c, several CG are surrounded by a handful of UFG, which have very thin and intermittent layers. This is called bimodal three. Meanwhile, as shown in Fig. 1d, the grain size of UFG and CG slightly increased. CG was only wrapped by a small fraction of UFG in a thin and discontinuous layer. This is called bimodal four. CG structure is enclosed in a three-dimensional network of UFG structure. Morphologically, this network of the UFG areas appears as "walls" that improve the structure stability. From the EBSD images, we also could observe growth twins, which will be analyzed in the discussion.

The above microstructural characteristics are further confirmed in Fig. 2. An adequate description of the grain size distribution and schematic illustration of grain size of UFG and CG in four bimodal structures are illustrated in Figs. 2a, 2c, 2e, and 2g. Meanwhile, the area fraction distributions determined through EBSD are shown in Figs. $2 \mathrm{~b}$, $2 \mathrm{~d}, 2 \mathrm{f}$, and $2 \mathrm{~h}$. The histograms of the grain size distribution have two distinct peaks quite similar to the hump, and this is the paramount feature that could be used to determine whether a grain size distribution is bimodal (Figs. 2a, 2c, 2e, and $2 \mathrm{~g}$ ) or unimodal. The histograms unambiguously indicate a bimodal grain size distribution with one peak in fine-grain area $(<1 \mu \mathrm{m})$ and another in coarse-grain area $(>1 \mu \mathrm{m})$. From bimodal one to bimodal four, the former peak corresponds to UFG, whose grain size increased from $0.45 \mu \mathrm{m}$ to $0.95 \mu \mathrm{m}$. CG related to the latter, whose grain size increased from $1.15 \mu \mathrm{m}$ to $3.05 \mu \mathrm{m}$. In bimodal one (Figs. 2a and 2b), the amount of UFG is high, totaling at $76.7 \%$, and area fraction is $29.8 \%$; hence, UFG can adequately wrap CG thickly. The grain size ranged from $0.35 \mu \mathrm{m}$ to $6.75 \mu \mathrm{m}$. By contrast, the amount of the UFG in other structures is far less than in bimodal one. As shown in Figs. 2c and 2d, UFG accounted for $63.5 \%$ of number fraction and $12.6 \%$ in total area, the grain size increasing from $0.15 \mu \mathrm{m}$ to $7.55 \mu \mathrm{m}$. In bimodal three (Figs. 2e and 2f), the grain size distribution is relatively average. In addition, UFG only accounted for $36.1 \%$ of the number fraction and area percentage is $4.5 \%$. The grain size of UFG ranged from $0.25 \mu \mathrm{m}$ to $0.95 \mu \mathrm{m}$ and that of CG ranged from $1.05 \mu \mathrm{m}$ to $7.95 \mu \mathrm{m}$. Bimodal four (Figs. $2 \mathrm{~g}$ and $2 \mathrm{~h}$ ) contains wide-sized grains, ranging from approximately $0.15 \mu \mathrm{m}$ to $14.15 \mu \mathrm{m}$. Although UFG accounts for $34.4 \%$ in the total number fraction and occupies $3.7 \%$ in area fraction, CG accounts for a larger percentage, resulting in CG surrounded by UFG in a thin layer similar to bimodal three. Note that although UFG are far more numerous, the area fraction of CG is always higher than UFG. The average grain sizes, all of which are calculated as an equivalent diameter derived from areal orientation measurements, are $0.90,1.30,2.30$, and $3.15 \mu \mathrm{m}$. Overall, the four bimodal structures possess a proximate feature; however, each has its own unique characteristics. The distinct characteristics of the grain size distribution were determined by UFG. From bimodal one to four, owing to the increasing grain size and the gradually decreasing area fraction of UFG, the degree of UFG layer surrounding the CG weakened, particularly result in the spatial distribution of UFG and CG was significantly different.

Fig. 3 shows the XRD patterns of four bimodal structures. Four samples all show two diffraction peaks located in $2 \theta=52.2^{\circ}$ and $61.1^{\circ}$. After comparison with the standard spectrum, two characteristic peaks of $\mathrm{Ni}$ (111) and (200) crystal planes [24] were noted, indicating that the prepared $\mathrm{Ni}$ has a complete crystal structure. In terms of relative intensity of diffraction peaks, bimodal one, bimodal three and bimodal four showed no preferred orientation. It also could be found that bimodal two present preferred orientation in (200).

\subsection{Mechanical behavior}

Fig. 4a shows the nominal stress-strain curves of bimodal Ni. In this work, we used UFG and CG Ni to illustrate our experimental result, or besides comparing the mechanical characteristics between unimodal $\mathrm{Ni}$ and bimodal Ni. All samples having the bimodal grain size distribution showed that the mechanical properties of A (bimodal one), B (bimodal two), C (bimodal three), and D (bimodal four) are quite different. The UTS and uniform elongation of curve $\mathrm{A}, \mathrm{B}, \mathrm{C}$, and $\mathrm{D}$ reach up to $847 \mathrm{MPa}$ and $13.1 \%, 792 \mathrm{MPa}$ and $12.8 \%, 697 \mathrm{MPa}$ and $11.6 \%$, and $569 \mathrm{MPa}$ and 19\%, respectively. In Curves $\mathrm{E}$ and $\mathrm{F}$ in Fig. 4a, which is for UFG Ni prepared by Krasilnikov et al. the material plastic instability was fleeting in a very small amount of deformation, exhibiting ultrahigh strength and low plasticity. Curve $\mathrm{E}\left(\mathrm{d}_{\text {grain }}=330 \mathrm{~nm}\right)$, and UTS reaching up to $1050 \mathrm{MPa}$, whereas the uniform elongation only reaches to $2 \%$. As also shown in Curve $\mathrm{F}\left(\mathrm{d}_{\text {grain }}=550 \mathrm{~nm}\right)$, the UFG Ni has high strength (UTS $=890 \mathrm{MPa}$ ), but low uniform elongation, only reaching to $3 \%$ [25]. Curve $\mathrm{G}$, which is a tensile engineering stress-strain curve of CG Ni displayed typical CG characteristics, that is, ultrahigh plasticity and low strength (UTS is $283 \mathrm{MPa}$ and uniform elongation reach to $31.8 \%$ ) [25]. Comparing with the UFG Ni and CG Ni, the uniform elongation and ultimate strength of bimodal Ni have been greatly improved. Interestingly, the tensile properties of the four bimodal structures varied widely and exhibit a very appealing combination of strength and ductility. Especially bimodal one whose structure is most stable could be regarded as a shell-and-core bimodal structure, UFG termed as "Shell" and an inner region (CG) which is termed as "Core".

Fig. 4b summarizes the strength-ductility trade-off for different grain size of $\mathrm{Ni}$ [23-26] (see the blue region), and this trend could be analogous to other metals as well [11-14]. Bimodal Ni stands out as an "out-of-the-box" exception with its high ductility and UFG-level strength, particularly for bimodal one. Both the strength and plasticity of the unimodal structure $\mathrm{Ni}$ are inferior to that of the bimodal structure. Elevating the strength of the material always compromises the ductility because the structural refinement leaves no room to store the dislocations. This phenomenon causes the dislocations readily annihilate into the abundant grain boundaries. Bimodal structure can be considered as an "artificial composite" composed of two phases possessing identical modulus of elasticity and different strengths. These features make bimodal materials have excellent comprehensive mechanical properties [27].

Photographs a, b, c, and d, which corresponds to bimodal one, two, three, and four, show the SEM observation of fracture surface morphologies of the bimodal Ni. All the photographs show the typical ductile fracture morphology with specific dimples having extended edges. In Fig. 5a, we can clearly see that some big dimples embedded in the flat surface are wrapped by several, shallow diminutive dimples. In Figs. 5 b, 5c, and 5d, dimple size increased significantly. Moreover, as shown in Fig. 5d, several small dimples encircle some bigger and deeper dimples. Although the dimple sizes are much larger than the grain size, 

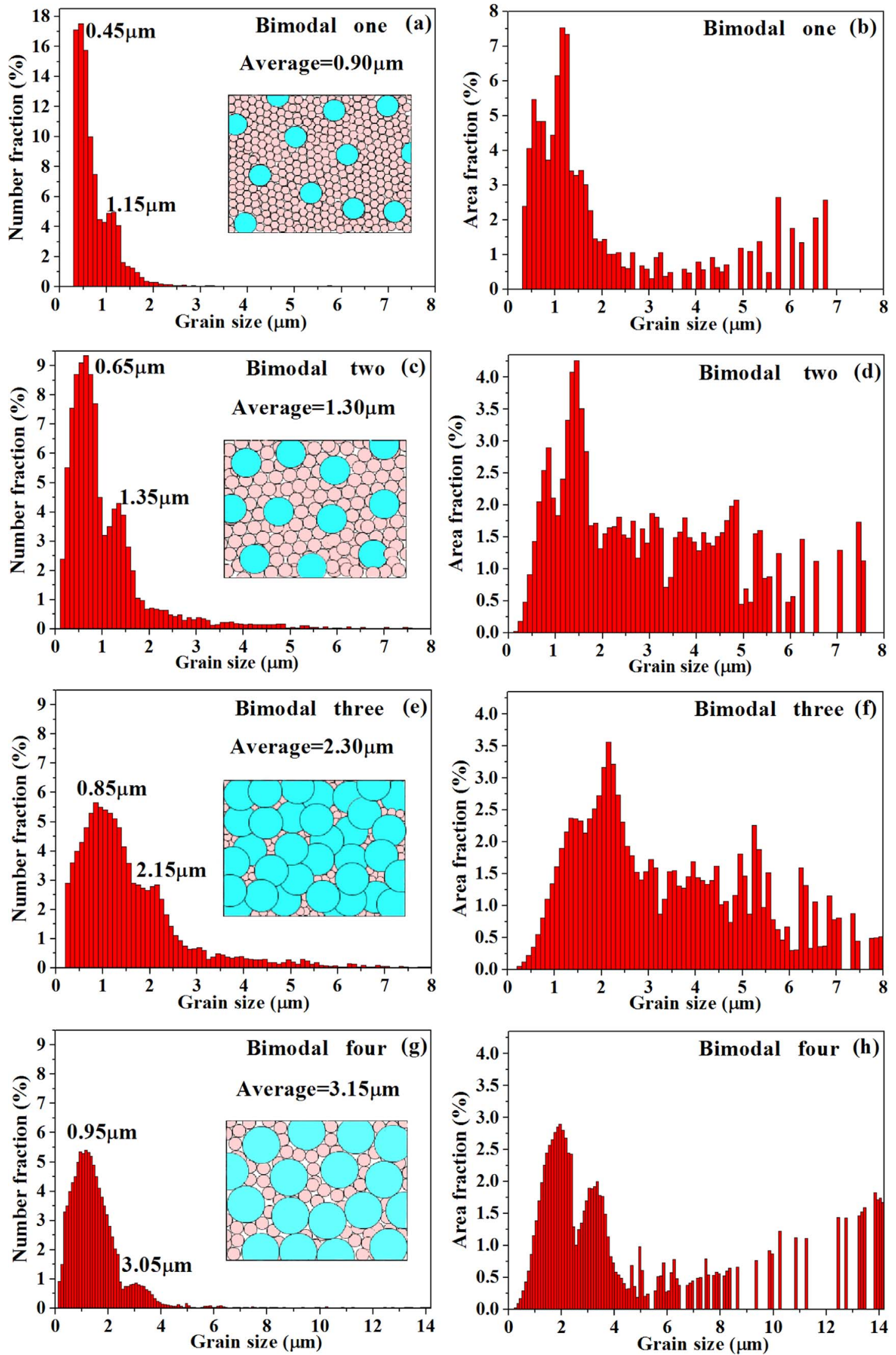

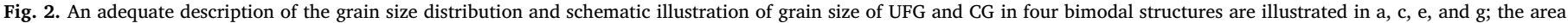
fraction distributions determined through EBSD are shown in b, d, f, and h. 


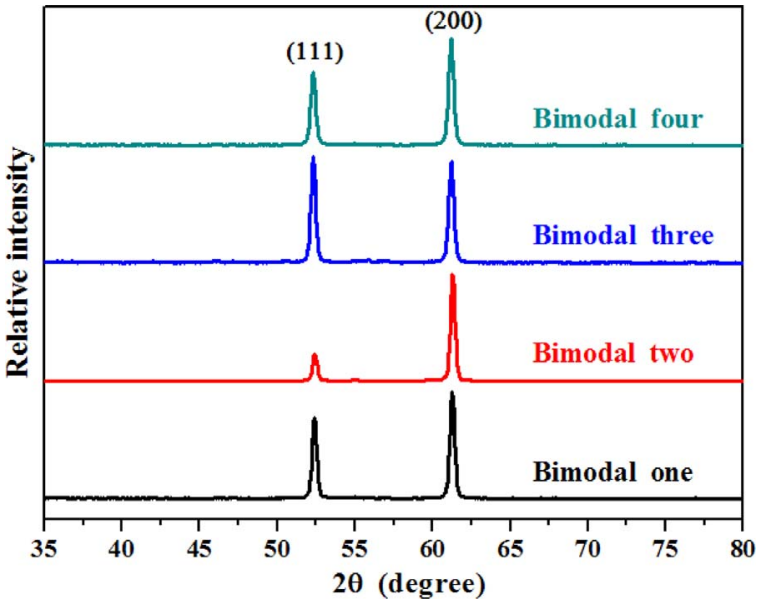

Fig. 3. XRD patterns of the four bimodal structures.
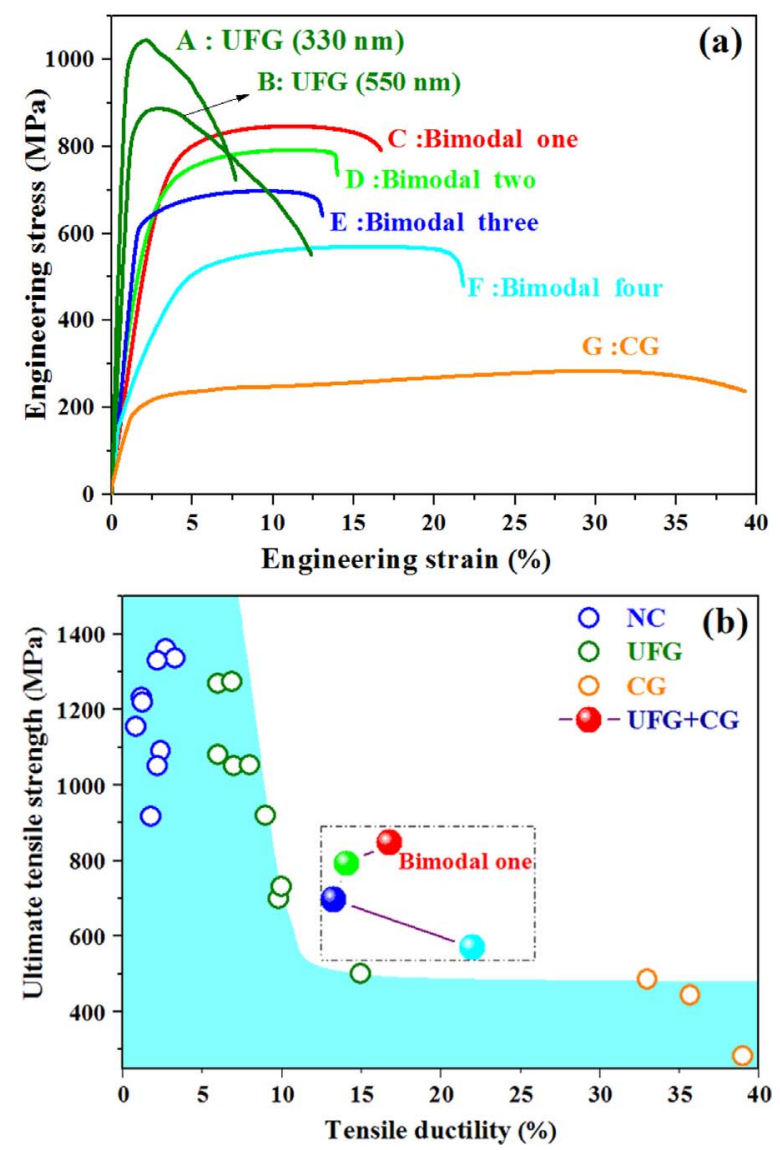

Fig. 4. (a) Tensile stress-strain curves of Ni; (b) the strength-ductility trade-off for different grain size of $\mathrm{Ni}$.

fine dimples are related to the UFG regions, and coarse dimples are related to the CG regions. The dimple morphology always corresponds to the grain size and distribution.

\section{Discussion}

\subsection{The effect of the microstructural features on the microhardness}

Based on the existing experimental results, the relationship between microstructure and mechanical behavior is further discussed by hardness testing. Microstructural features of bimodal structures are reflected in the microhardness variation in Fig. 6. Fifteen hardness values of bimodal structures were tested respectively. The distribution of hardness values in bimodal one (280.7 HV $311.7 \mathrm{HV})$ and bimodal three (252.6 HV $294.7 \mathrm{HV}$ ) is relatively narrow. Yet, the range of hardness variation of bimodal two $(249.2 \mathrm{HV} \sim 307.8 \mathrm{HV})$ and bimodal four (210.4 HV $296.3 \mathrm{HV}$ ) is widely, as shown in Fig. 6. Hardness values and hardness variation correlate with the grain size, occupied ratio of the UFG and CG areas. In Fig. 1a, it is found that UFG occupies a higher proportion fraction, and the grain size distribution is concentrated on the smaller grain determining the hardness values of bimodal one concentrated on upper positions. From Fig. 1d, it could be discovered that bigger CG occupies a higher proportion fraction and the grain size distribution range is relatively wide, which result in hardness variation widely and the hardness values concentrated on lower positions. Microstructure of specimens can be reflected by the hardness characteristics. As anticipated, the average Vickers values of four bimodal structures are 291.5, 279.8, 273.1, and 243.9 HV. As average grain size increases, hardness decreases. Microhardness measurement results agree reasonably well with the tensile data showing a consistent trend of strength.

\subsection{Strengthening and toughening mechanism}

Normalized work-hardening rate $\Theta$ is defined by the following Equation: $\Theta=1 / \sigma(\partial \sigma / \partial \varepsilon)_{\varepsilon}$, where $\sigma$ is true stress and $\varepsilon$ is true strain. Fig. 7 shows curves of normalized strain-hardening rate $(\Theta)$ against true strain about UFG, CG and four kinds of bimodal structure. At the beginning of the deformation (true strain $\leq 2.6 \%$ ), bimodal structures display more positive strain hardening to meaningful strains than unimodal structure of UFG and CG. Particularly bimodal one, it forms a special shell-and-core structure, whose work-hardening rate is the highest. In order to do deeper research on the mechanism of tensile deformation of this structure, its orientation map is discussed in Fig. 8. Fig. 8 shows the grain boundaries distribution including growth twin boundaries. Growth twins in this article are referred to $\Sigma 3(111)$ coherent twins, shown in red lines and other boundaries in black lines. Coherent twin boundaries have a symmetrical structure and low-energy configuration, strengthening effect follows the empirical H-P relation, could also refine the microstructure, and keep material in high strainhardening coefficients [28-31]. As shown in Fig. 8, abundant coherent twin boundaries distribute in UFG.

Deformation initial, as a result of small population of CG surrounded by quite a lot of UFG and a large number of $\Sigma 3(111)$ coherent twins existing in UFG, UFG areas can be visualized as skeleton of the microstructure. Hence, material cannot be plastically deformed without achieving deformation in UFG areas. Interconnected UFG and $\Sigma 3(111)$ coherent twins become the high stress area leading to higher workhardening rate and yield strength.

As strain continues to increase, yield takes place in the UFG areas firstly. As soft CG areas is constrained by the hard UFG. Subsequently, the plastic deformation of UFG areas brings about deforms to these CG. Plastic strain is transferred to soft CG regions through the interface of CG/UFG areas. The plastic deformation occurs in CG and generating the strain hardening, which is contributed to increased strength with increasing straining. It appears that this unique structure and exist of

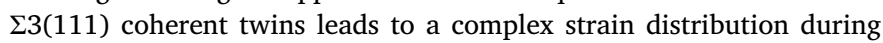
plastic deformation to keeps the higher strength and effective delays plastic instability of the material.

\section{Summary}

An electrodeposition technique prepared four types of bimodal Ni having a huge disparity in mechanical properties, attributed to grain size and spatial distribution of CG and UFG areas. Among them, bimodal one with a grain size of $0.45 \mu \mathrm{m}$ in UFG regions and $1.15 \mu \mathrm{m}$ in CG regions has the best comprehensive mechanical properties. In other words, when micrometer-sized grains were embedded in UFG matrix, 

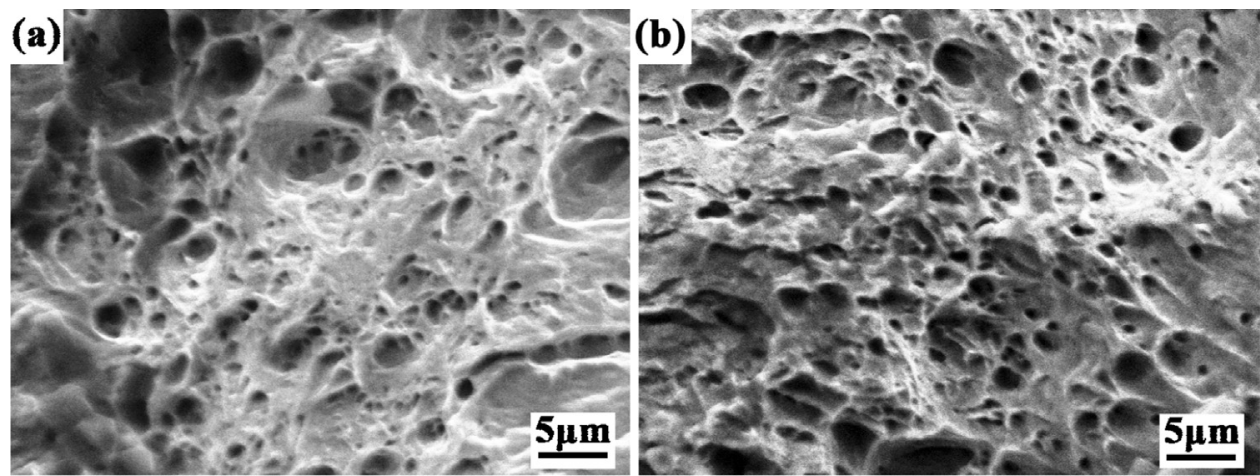

Fig. 5. Show the SEM observation of fracture surface morphologies of the bulk bimodal Ni.
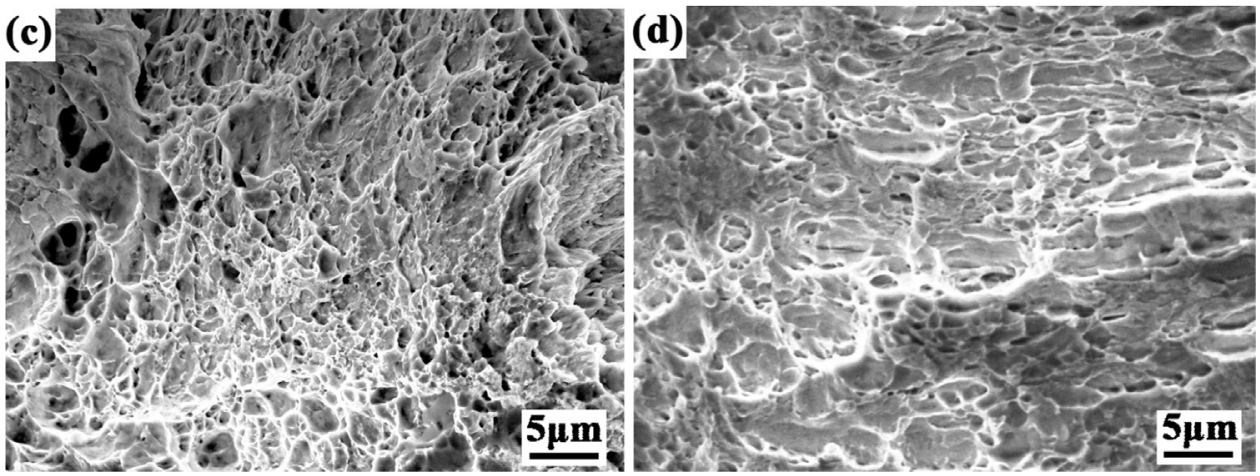

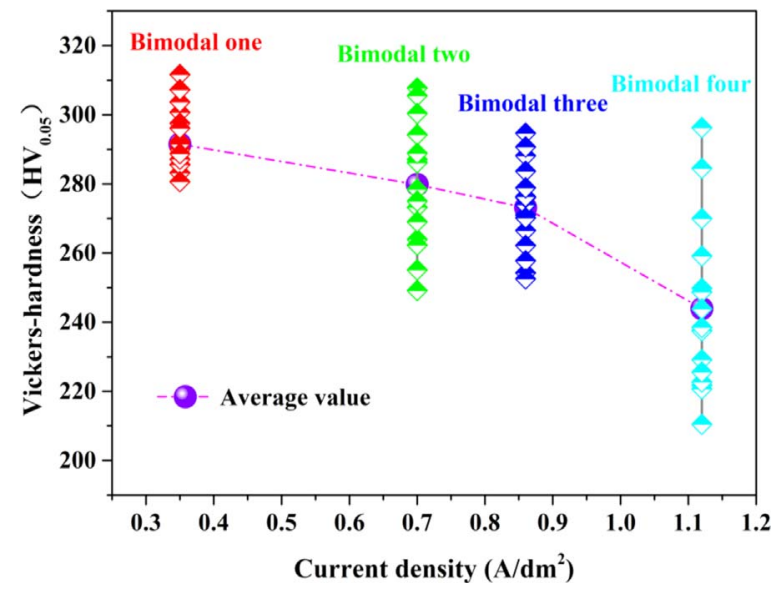

Fig. 6. Microhardness variation and average microhardness of four bimodal structures.

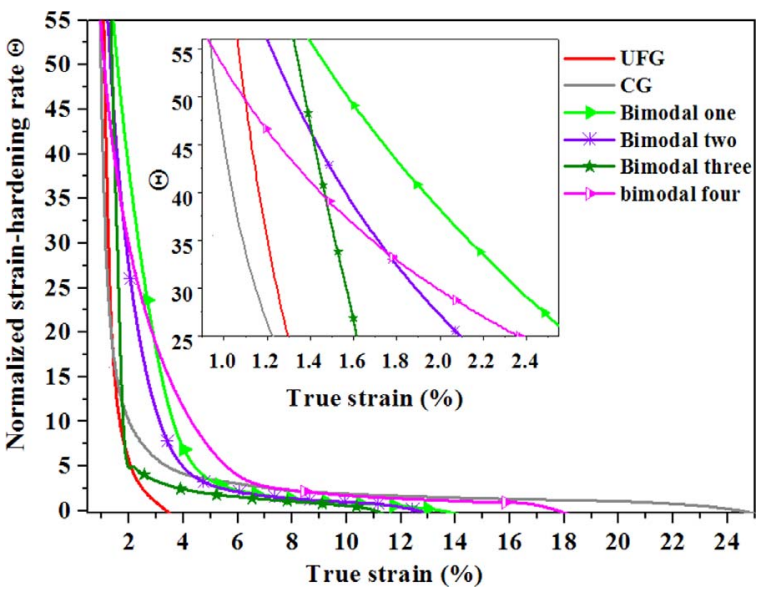

Fig. 7. Normalized strain-hardening rate $(\Theta)$ against true strain.

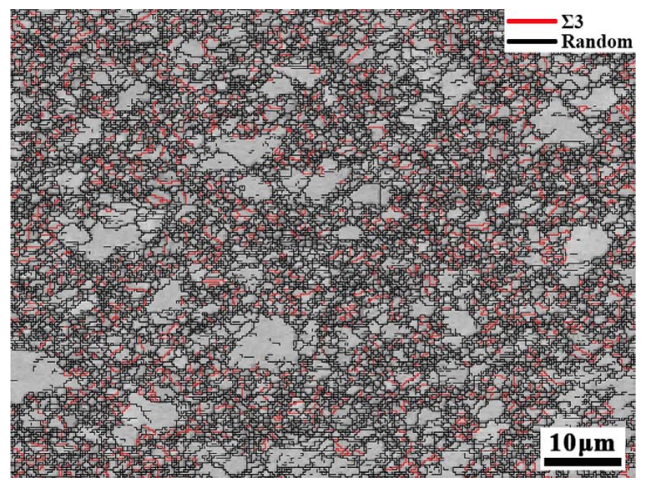

Fig. 8. Orientation map from bimodal one. $\Sigma 3(111)$ coherent twin boundaries are shown in red lines and other boundaries in black lines. (For interpretation of the references to color in this figure legend, the reader is referred to the web version of this article.)

bimodal Ni achieved better strength-to-ductility ratio. Deformation initial, bimodal structures display more positive strain hardening to meaningful strains than unimodal structure of UFG and CG, particularly for bimodal one, work-hardening rate of bimodal one is the highest thanks to its unique shell-and-core structure and a large number of

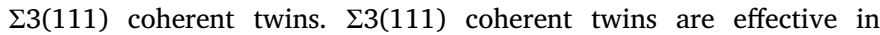
blocking dislocation motions and improving work-hardening ability, imparting an ultrahigh tensile strength and a considerable ductility.

\section{Acknowledgements}

This research was supported by the National Natural Science Foundation of China (Grant No. 51371123), the National Natural Science Foundation of Shanxi Province (Grant No. 2014011002) and the Research Fund for the Doctoral Program of Higher Education of China (20131402110003).

\section{References}

[1] K. Lu, L. Lu, S. Suresh, Strengthening materials by engineering coherent internal boundaries at the nanoscale, Science 324 (2009) 349-352. 
[2] D. Jang, X. Li, H. Gao, J.R. Greer, Deformation mechanisms in nanotwinned metal nanopillars, Nat. Nanotechnol. 7 (2012) 594-601.

[3] Y. Wang, M. Chen, F. Zhou, E. Ma, High tensile ductility in a nanostructured metal, Nature 419 (2002) 912-915.

[4] L. Lu, Y. Shen, X. Chen, L. Qian, K. Lu, Ultrahigh strength and high electrical conductivity in copper, Science 304 (2004) 422-426.

[5] K.M. Youssef, R.O. Scattergood, K.L. Murty, J.A. Horton, C.C. Koch, Ultrahigh strength and high ductility of bulk nanocrystalline copper, Appl. Phys. Lett. 87 (2005) (091904-091904-3).

[6] R.Z. Valiev, I.V. Alexandrova, Y.T. Zhu, T.C. Lowe, Paradox of strength and ductility in metals processed by severe plastic deformation, J. Mater. Res. 17 (2002) 5-8.

[7] Y.H. Zhao, T. Topping, J.F. Bingert, High tensile ductility and strength in bulk nanostructured nickel, Adv. Mater. 20 (2008) 3028-3033.

[8] T.H. Fang, W.L. Li, N.R. Tao, K. Lu, Revealing extraordinary intrinsic tensile plasticity in gradient nano-grained copper, Science 331 (2011) 1587-1590.

[9] X.L. Wu, P. Jiang, L. Chen, F.P. Yuan, Y.T. Zhu, Extraordinary strain hardening by gradient structure, Proc. Natl. Acad. Sci. USA 111 (2014) 7197-7201.

[10] Y.J. Wei, Y.Q. Li, L.C. Zhu, Evading the strength-ductility trade-off dilemma in steel through gradient hierarchical nanotwins, Nat. Commun. 5 (2011).

[11] X.X. Huang, N. Hansen, N. Tsuji, Hardening by annealing and softening by deformation in nanostructured metals, Science 312 (2006) 249-251.

[12] V. Ruslan, Nanostructuring of metals by severe plastic deformation for advanced properties, Nat. Mater. 3 (2004) 511-516.

[13] Y.T. Zhu, X.Z. Liao, Nanostructured metals-retaining ductility, Nat. Mater. 3 (2004) 351-352.

[14] E. Ma, Eight routes to improve the tensile ductility of bulk nanostructured metals and alloys, JOM 58 (2006) 49-53.

[15] Y.T. Zhu, T.G. Langdon, The fundamentals of nanostructured materials processed by severe plastic deformation, JOM 56 (2004) 58-63.

[16] M.A. Meyers, A. Mishra, D.J. Benson, Mechanical properties of nanocrystalline materials, Prog. Mater. Sci. 51 (2006) 427-556.

[17] J.R. Weertman, Nanostructured Materials: Proceeding, Properties and Applications, William Andrews Norwich, New York, 2002.

[18] X.L. Wu, F.P. Yuan, M.X. Yang, Nanodomained nickel unite nanocrystal strength with coarse-grain ductility, Sci. Rep. 5 (2015) 11728
[19] N. Krasilnikov, W. Lojkowski, Z. Pakiela, Tensile strength and ductility of ultra-finegrained nickel processed by severe plastic deformation, Mater. Sci. Eng. A 397 (2005) 330-337.

[20] S. Berbenni, V. Favier, M. Berveiller, Impact of the grain size distribution on the yield stress of heterogeneous materials, Int. J. Plast. 23 (2007) 114-142.

[21] M. Yadollahpour, H. Hosseini-Toudeshky, Material properties and failure prediction of ultrafine grained materials with bimodal grain size distribution, Eng. Comput.Ger. 33 (2017) 125-136.

[22] H.E. Sabzia, A.Z. Hanzakia, H.R. Abedia, The effects of bimodal grain size distributions on the work hardening behavior of a TRansformation-TWinning induced plasticity steel, Mater. Sci. Eng. A. 678 (2016) 23-32.

[23] Q. Tao, K. Ibrahim, M. Michael, Mechanical properties of nanocrystalline and ultrafine-grained nickel with bimodal microstructure, Adv. Eng. Mater. 16 (2014) 1323-1339.

[24] B.D. Cullity, Elements of X-ray Diffraction, second ed., Addison-Wesley Reading (MA), New York, 1978

[25] N. Krasilnikov, W. Lojkowski, Z. Pakiela, Tensile strength and ductility of ultra-finegrained nickel processed by severe plastic deformation, Mater. Sci. Eng. A. 397 (2005) 330-337.

[26] F. Ebrahimi, G.R. Bourne, M.S. Kelly, T.E. Matthews, Mechanical properties of nanocrystalline nickel produced by electrodeposition, Nanostruct. Mater. 11 (1999) 343-350.

[27] D. Orlov, H. Fujiwara, K. Ameyama, Obtaining copper with harmonic structure for the optimal balance of structure-performance relationship, Mater. Trans. 54 (2013) 1549-1553.

[28] Y.F. Shen, L. Lu, Q.H. Lu, Z.H. Jin, K. Lu, Tensile properties of copper with nanoscale twins, Scr. Mater. 52 (2005) 989-994.

[29] X. Zhang, A. Misra, H. Wang, A.L. Lima, M.F. Hundley, R.G. Hoagland, Effects of deposition parameters on residual stresses, hardness and electrical resistivity of nanoscale twinned 330 stainless steel thin films, J. Appl. Phys. 97 (2005) 1-5.

[30] T. Zhu, J. Li, A. Samanta, H.G. Kim, S. Suresh, Interfacial plasticity governs strain rate sensitivity and ductility in nanostructured metals, Proc. Natl. Acad. Sci. USA 104 (2007) 3031-3036.

[31] K.A. Afanasyev, F. Sansoz, Strengthening in gold nanopillars with nanoscale twins, Nano Lett. 7 (2007) 2056-2062. 